\title{
ON THE EXISTENCE OF COMPLETE PARALLEL VECTOR FIELDS
}

\author{
DAVID J. WELSH, JR.
}

\begin{abstract}
In 1966, Chern asked which compact orientable manifolds carry a Riemannian metric and a vector field which is parallel under this metric. An earlier paper answered this without assuming orientability. The present paper applies techniques which work without compactness to identify manifolds which can carry complete parallel vector fields
\end{abstract}

1. Introduction. Compact manifolds which admit a parallel vector field with respect to some metric have been characterized in a previous paper [6]. Namely, they are the (compact) fibre bundles over tori with finite structural group. The dimension of the torus can be assumed to be the number of linearly independent parallel vector fields. This answered a question of Chern [1].

The proof in [6] used the Albanese map and some well-known facts about harmonic 1-forms. These tools are not available in the noncompact case, and, in fact, the noncompact case has apparently not even been touched. The aim of this paper is to develop tools that can be used in a noncompact setting.

In particular, a theorem about the existence of complete parallel vector fields is proven for a class of manifolds which includes (but is not restricted to) those whose first integral homology class is finitely generated. If $M$ is not a product $\mathbf{R} \times M^{\prime}$, then the following are equivalent:

(i) $M$ carries a complete parallel vector field under some metric.

(ii) There is a circle action on $M$ whose orbits are not real homologous to zero.

(iii) $M$ is a fibre bundle over a circle with finite structural group.

Note that $\mathbf{R} \times M^{\prime}$ always admits a complete parallel vector field with respect to a suitable metric.

2. Killing vector fields on noncompact manifolds. The isometry group of a compact manifold is always compact. For more general $M$, the identity component of the isometry group $I_{0}(M)$ is compact when $M$ is not diffeomorphic to the product of a Euclidean space with some other manifold [7, 4]. This fact simplifies the following exposition.

For the rest of this section, $M$ will be a Riemannian manifold that carries a complete parallel vector field $v$. The case that $M$ is a product $\mathbf{R} \times M_{1}$ will be excluded so that $I_{0}(M)$ will be compact. The Lie algebra of $I_{0}(M)$ will be identified with that of the complete Killing vector fields on $M$.

Received by the editors October 31, 1984 and, in revised form, May 24, 1985.

1980 Mathematics Subject Classification. Primary 53C05; Secondary 53C65, 58A10.

(C)1986 American Mathematical Society $0002-9939 / 86 \$ 1.00+\$ .25$ per page 
Let $C$ be the closure of the 1-parameter subgroup generated by $v$ in $I_{0}(M)$. It is compact and abelian, hence a torus. Denote by exp the exponential map on $C$. Then $\exp ^{-1}(0)$, where 0 is the identity of $C$, is a lattice that spans the Lie algebra of $C$. There is an element $w$ in $\exp ^{-1}(0)$ that is not orthogonal to $v$ at some point of $M$.

Note that the inner product $\langle v, w\rangle$ is constant on each orbit of $C$, since $C$ is compact (see e.g. [8]). On the other hand, if $X$ is a vector orthogonal to an orbit of $C$, then

$$
X\langle v, w\rangle=\left\langle\nabla_{X} v, w\right\rangle+\left\langle v, \nabla_{X} w\right\rangle=-\left\langle\nabla_{v} w, X\right\rangle=-\left\langle\nabla_{w} v, X\right\rangle-\langle[v, w], X\rangle=0 .
$$

Hence $\langle v, w\rangle$ is constant.

The 1-parameter subgroup generated by $w$ is a circle. Integration of the dual form to $v$ along an orbit of the subgroup yields a nonzero result. Thus the following is proven:

PROPOSITION 2.1. If a Riemannian manifold $M$ admits a complete parallel vector field, then either $M$ is diffeomorphic to the product of a Euclidean space with some other manifold, or else there is a circle action on $M$ whose orbits are not real homologous to zero.

3. Construction of the bundle. This section is devoted to proving the following theorem:

THEOREM 3.1. Let $M$ be a differentiable manifold such that $H_{1}(M, Z)$ is the direct sum of a free abelian group and a torsion group. If $M$ admits a smooth $S^{1}$-action for which some orbit is not torsion in homology, then $M$ is a fibre bundle over a circle with finite structural group.

The condition on the homology group, in conjunction with the deRham Theorem, insures the existence of an integral closed 1 -form $\theta$ that yields a nonzero result when evaluated on orbits. Choose $\theta$ to be invariant under the $S^{1}$-action. One can now define a map similar to the Albanese map for compact manifolds.

Namely, fix $e$ in $M$. Then for any piecewise differentiable path $\eta$ on $M$, the number $\int_{\eta} \theta \bmod \mathbf{Z}$ depends only on the endpoints of $\eta$ since $\theta$ is an integral form. By requiring $\eta(0)$ to always be $e$, a map from $M$ into the circle $T=\mathbf{R} / \mathbf{Z}$ is defined by setting $\phi(\eta(0))=\left[\int_{\eta} \theta\right]$, where the brackets signify that the result is to be taken modulo $\mathbf{Z}$. The expression $\int_{\eta(0)}^{\eta(1)} \theta$ may be used for $\int_{\eta} \theta$.

The map $\phi$ is differentiable. Indeed, if $\eta$ is defined on $[0,1+\varepsilon)$, and is differentiable about $t=1$, then

$$
d /\left.d t\right|_{t=1} \int_{\eta} \theta=d /\left.d t\right|_{t=1} \int_{0}^{t} \eta^{*} \theta=\eta^{*} \theta(d / d t)_{1}=\theta(d \eta / d t)_{\eta(1)}
$$

If $S^{1}$ is considered as a 1-parameter group, then its orbits are naturally parameterized. If $\eta$ is such a parametrization of an orbit, then $\theta(d \eta / d t)$ is a nonzero constant. This means that $\phi$ is a submersion.

There is an induced map of $S^{1}$ into $T$ defined by $h(s)=\phi(s(e))$. This is differentiable of course, but even more is true. 
LeMMA 3.2. The map $h: S^{1} \rightarrow T$ is a homomorphism.

Proof.

$$
\begin{aligned}
h\left(s_{1} \circ s_{2}\right) & =\left[\int_{e}^{s_{1}\left(s_{2}((e))\right.} \theta\right]=\left[\int_{e}^{s_{2}(e)} \theta\right]+\left[\int_{s_{2}(e)}^{s_{1}\left(s_{2}(e)\right)} \theta\right] \\
& =h\left(s_{2}\right)+\left[\int_{e}^{s_{1}(e)} \theta\right]=h\left(s_{2}\right)+h\left(s_{1}\right) .
\end{aligned}
$$

This defines an action of $S^{1}$ on $T$, via $s(\phi(y))=h(s)+\phi(y)$, which is independent of the choice of $y$ in the fiber. Furthermore, the right-hand side becomes $\left[\int_{e}^{s e} \theta\right]+\left[\int_{e}^{y} \theta\right]=\left[\int_{e}^{s e} \theta+\int_{s e}^{s y} \theta\right]=\phi(s(y))$. Thus we have

LeMma 3.3. The function $\phi$ is equivariant with respect to the given actions of $S^{1}$.

RemarK. The map $h$ can be defined on any compact group $G$ acting on $M$. The lemmas remain true if $\theta$ is $G$-invariant.

The importance of this lemma is that $S^{1}$ preserves the fibers of $\phi$. In particular, the fibers are all diffeomorphic. Now let $F=\phi^{-1}([0])$, and define $K$ to be the kernel of $h$. Then $K \rightarrow S^{1} \rightarrow T$ is a principal bundle over $T$, where $K$ is a finite cyclie group. There is an associated bundle $F \rightarrow S^{1} \times{ }_{K} F \rightarrow T$, where $S^{1} \times_{K} F$ is the quotient space of the action on $S^{1} \times F$ given by $k(s, f)=\left(s \circ k^{-1}, k(f)\right), k \in K$.

The total space of the bundle is diffeomorphic to $M$. In fact, there is a commutative diagram

$$
\begin{gathered}
S^{1} \times F \stackrel{E}{\rightarrow} M \\
\pi \searrow \quad \nearrow \psi \\
S^{1} \times{ }_{K} F
\end{gathered}
$$

where $E$ is the evaluation map given by the group action, $\pi$ is the projection mapping, and $\psi$ is a diffeomorphism which preserves fibers.

4. Concluding remarks. In the rest of this paper, the statement that $M$ admits a parallel vector field means that $M$ carries one under a suitable metric.

It has already been shown in [6] that any fibre bundle over a torus with finite structural group admits a (complete) parallel vector field. For manifolds not of the form $\mathbf{R} \times M^{\prime}$, and satisfying the homology condition of Theorem 3.1, this demonstrates the equivalence of statements (i)-(iii) of the introduction.

A natural question is what happens if the condition on $H_{1}(M, \mathbf{Z})$ is dropped? A manifold for which $H_{1}(M, \mathbf{Z})=\mathbf{Q}$ certainly causes the proof to break down. In fact, $H^{1}(M, \mathbf{Z})=0$ even though $H^{1}(M, \mathbf{R})=\mathbf{R}$. On the other hand, let $\phi: M \rightarrow S^{1}$ be a fibration as above. Then $M$ is finitely covered by $S^{1} \times F$. The composition $H^{1}\left(S^{1}\right)$ $\rightarrow H^{1}(M) \rightarrow H^{1}\left(S^{1} \times F\right)$ is injective, and hence $H^{1}(M, \mathbf{Z}) \neq 0$. Thus such a fibration cannot even exist on this manifold.

In fact, the property $H^{1}(M, Z) \neq 0$ implies that the first homology group must have a free factor. Can a complete parallel vector field exist on a manifold for which $H^{1}(M, Z)=0$ ? An answer to this question should indicate whether the homology condition is actually needed. 
It should be noted that Percell has shown that any compact manifold with boundary admits a parallel vector field [3]. Omitting the boundary yields a noncompact manifold that carries an incomplete parallel vector field.

\section{REFERENCES}

1. S. S. Chern, Geometry of G-structures, Bull. Amer. Math. Soc. 72 (1966), 167-219.

2. S. Kobayashi and K. Nomizu, Foundations of differential geometry, Vols. 1 and 2, Interscience, New York, 1963, 1969.

3. Peter Percell, Parallel vector fields on manifolds with boundary, J. Differential Geom. 16 (1981), 101-104.

4. S. Stranzalos, Dynamische systeme and topologische aktionen, Manuscripta Math. 13 (1974), 207-211.

5. Frank W. Warner, Foundations of differential manifolds and Lie groups, Scott-Foresman, Glenview, Ill., 1971.

6. David J. Welsh, Jr., Manifolds that admit parallel vector fields, Illinois J. Math. 30 (1986), 9-18.

7. Shing Tung Yau, Remarks on the group of isometries of a Riemannian manifold, Topology 16 (1977), 239-247.

8. K. Yano and S. Bochner, Curvature and Betti numbers, Ann. of Math. Studies, no. 32, Princeton Univ. Press, Princeton, N. J., 1953.

Department of Mathematics, University of Missouri - St. Louis, 8001 Natural Bridge Road, ST. Louis, MisSOURI 63121 